\title{
(3) Pathophysiology of Idiopathic Portal Hypertension
}

\author{
Haruo KAMEDA and Tadanao TAKEDA \\ The Second Department of Internal Medicine, Faculty of Medicine, \\ University of Tokyo
}

Idiopathic portal hypertension is clinically characterized by the elevated portal pressure with marked splenomegaly, anemia, no evidence of extrahepatic obstruction of the portal vein and absence of cirrhosis. Etiology and pathogenesis of the disease still remains obscure. In the present study, pathologic physiology of idiopathic portal hypertension was investigated with special emphasis on hemodynamic aspects including splenic, portal and hepatic arterial circulation.

\section{Materials and Methods}

Eighty cases of idiopathic portal hypertension, who had been hospitalized in the Second Department of Internal Medicine, University of Tokyo, were analyzed. Among them, 39 cases were operated upon and diagnosis was confirmed. Detailed hemodynamic studies were, for the most part, made on these surgical cases. For comparison, 25 cases of cirrhosis with splenomegaly and 41 cases of cirrhosis without marked splenomegaly were analyzed. Patients without any demonstrable hepatosplenic disease who were subjected to hemodynamic or visceral angiographic studies served as controls. In most patients, diagnosis was made on not only clinical, radiological, peritoneoscopic findings but also histological examinations by wedge or needle biopsy.

Hemodynamic studies included hepatic venous catheterization, selective serial celiac arteriography and indirect portography, estimation of hepatic blood flow utilizing colloidal ${ }^{198} \mathrm{Au}$ clearance, counting of radioactivity and scintiscanning of organs following ${ }^{131}$ I-MAA injected into the celiac trunk and/or into the spleen, estimation of splenic blood flow by determination of the washout curve of splenic radioactivity using ${ }^{85} \mathrm{Kr}$ injected into the celiac artery. Portal venous pressure was determined at operation and direct portography was made in some operated cases.

\section{Results}

Wedged hepatic venous pressure in patients with idiopathic portal hypertension ranged from 104 to $265 \mathrm{~mm}$. saline.

Although in some cases in the advanced stage of the disease marked collateral channels were demonstrated by portography and histological examination of the liver revealed marked periportal fibrosis, majority of the cases showed slight to moderate fibrotic changes in the liver. 
Selective serial celiac arteriography in patients with idiopathic portal hypertension revealed a large dilated splenic artery (mean caliber $7.6 \mathrm{~mm}$. as compared with 5.5 . $\mathrm{mm}$. in controls and cirrhosis) and a huge splenic silhouette. The common and proper hepatic arteries were shown to be rather small in their caliber (mean caliber of the proper hepatic artery $2.9 \mathrm{~mm}$. as compared with $4.7 \mathrm{~mm}$. in controls) and relatively straightened in their course. The peripheral arterial vascularity appeared to be attenuated. In veno-portal phase, the large patent splenic and portal veins were visualized and relatively good opacification of peripheral intrahepatic portal branches.

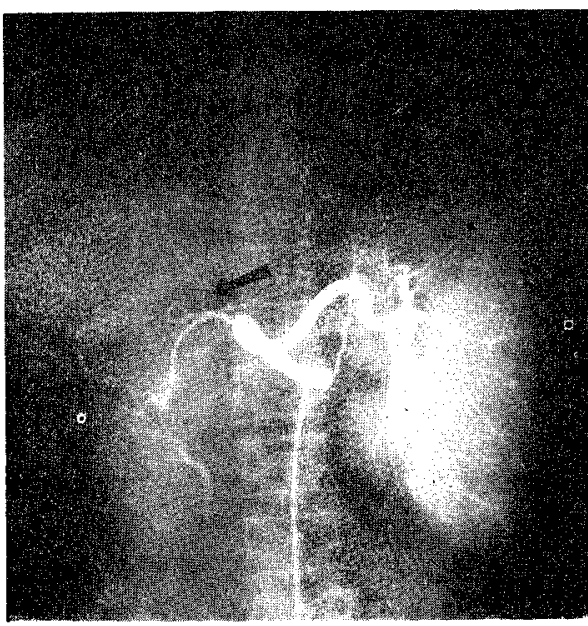

A. Arterial phase

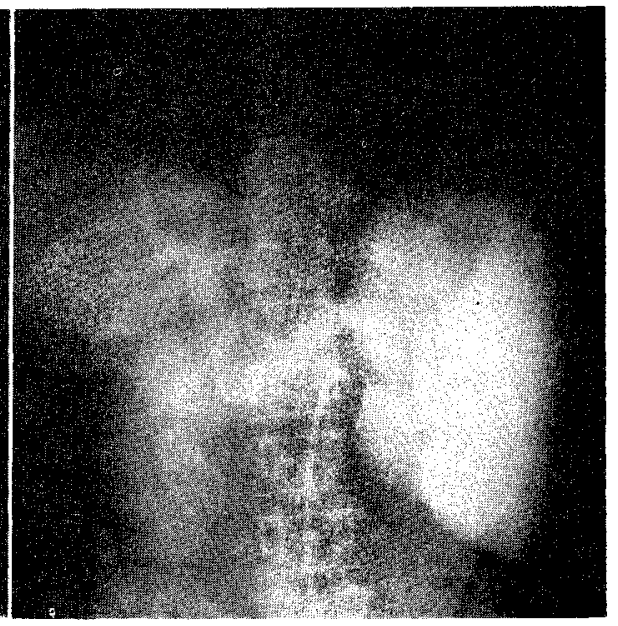

B. Venous phase

Fig. 1. Selective celiac arteriographic finding of idiopathic portal hypertension.

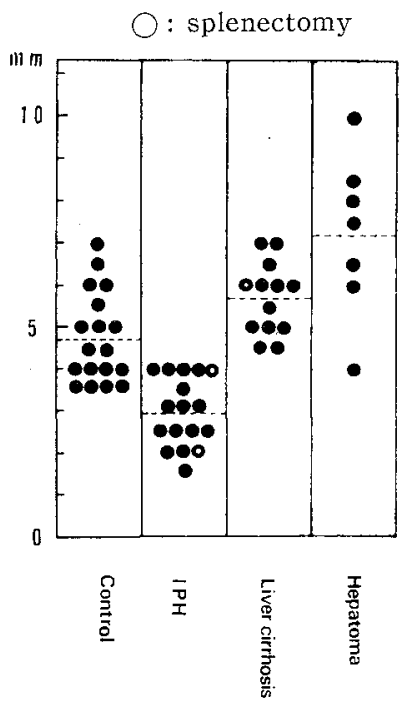

Fig. 2. Caliber of the proper hepatic artery.

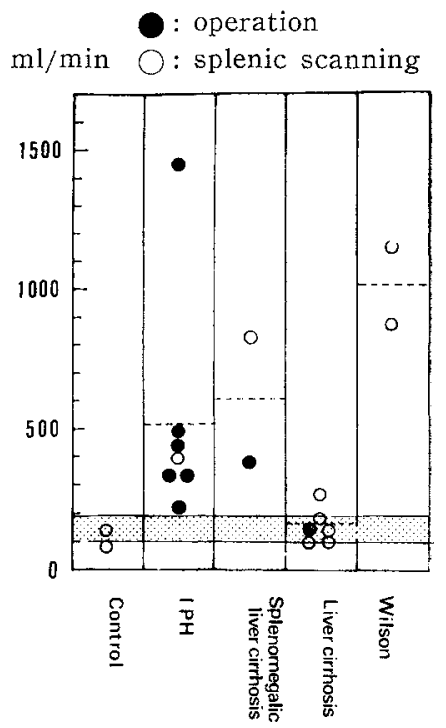

Fig. 3. Total splenic blood flow. 
were obtained (Fig. 1, A and B). The appearance time of contrast media in the splenic and portal veins was not delayed. These findings made a remarkable contrast to those in patients with cirrhosis of the liver. The common and proper hepatic arteries in cirrhosis were visualized with tortuousity and increased in their caliber (mean caliber of the proper hepatic artery $5.7 \mathrm{~mm}$.) (Fig. 2). The appearance of venous visualization was delayed in cirrhosis with poor opacification of the portal system.

Splenic blood flow per unit weight of the spleen measured with ${ }^{85} \mathrm{Kr}$ washout technique gave almost normal values (approximately $110 \mathrm{ml} . / 100 \mathrm{~g} . / \mathrm{min}$.) in idiopathic portal hypertension as well as in cirrhosis with marked splenomegaly, while in cirrhosis without splenomegaly, reduced values were obtained $(66 \mathrm{ml} . / 100 \mathrm{~g} . / \mathrm{min}$. on an average). When the total splenic blood flow was calculated on the basis of the estimated splenic weight, marked increase of flow amounting to 2 to 5 times as much as normal was evident'(Fig. 3). Significant negative correlation was found between splenic blood flow per unit weight of tissue and appearance time of the splenic vein serial angiography.

$\mathrm{K}$ values from colloidal ${ }^{198} \mathrm{Au}$ clearance technique, which are indicative of hepatic blood flow, were within almost normal range in patients with idiopathic portal hypertension, whereas they were considerably reduced in cirrhotic patients. This is in agreement with results obtained by the BSP method.

Distribution of arterial blood supply was studied by scintiscanning of the liver and spleen following intraarterial injection of ${ }^{131}$ I-MAA into the celiac artery. In cirrhosis of the liver, high radioactivity was demonstrated over the hepatic region, indicating a rich arterial supply to the liver through the celiac axis. On the other hand, in idiopathic portal hypertension, activity was very scanty over the hepatic area, while a bulk of radioactivity was detected over the spleen. The results indicate that the majority of the arterial blood is shared by the spleen and hepatic arterial perfusion is much reduced in idiopathic portal hypertension.

The amount of blood shunted to the lung was estimated by pulmonary scan following intrasplenic injection of ${ }^{131}$ I-MAA. Relatively less blood bypassed the liver in idiopathic portal hypertension as compared in cirrhosis. Significant intrahepatic shunt which was usually observed in cirrhosis by means of counting radioactivity over the hepatic area continuously after injection could not be demonstrated in idiopathic portal hypertension. It is assumed that a large portion of blood passing through the spleen comes into the liver in the latter condition.

\section{Conclusions}

Angiographic and hemodynamic studies on idiopathic portal hypertension revealed following characteristics of the disease: 1) increased splenic blood flow, 2) decreased arterial blood supply to the liver, 3) development of extrahepatic shunt, but poor intrahepatic shunt, and 4) no evidence of extrahepatic portal obstruction. 